\title{
Corticotropinoma as the underlying cause of intermittent Cushing's syndrome in a patient previously diagnosed with primary pigmented nodular adrenocortical disease
}

\author{
Agnieszka tebek-Szatańska', Maria Stelmachowska-Banaś', Grzegorz Zieliński², Andrzej Styk², \\ Karolina M. Nowak', Lucyna Papierska ${ }^{1}$ \\ ${ }^{1}$ Department of Endocrinology, Centre of Postgraduate Medical Education, Warsaw, Poland \\ ${ }^{2}$ Department of Neurosurgery, Military Institute of Medicine, Warsaw, Poland
}

Key words: corticotropinoma; Cushing's syndrome; Carney complex; PPNAD

A 32-year-old female with a 10-year history of symptoms suggesting intermittent Cushing's syndrome (CS) and a negative family history of hypercortisolaemia was first admitted for endocrinological evaluation in 2010. She was diagnosed with adrenocorticotropin (ACTH)-independent CS with hyperandrogenism and left adrenal hyperplasia. She underwent unilateral adrenalectomy. Histopathological examination revealed primary pigmented nodular adrenocortical disease (PPNAD). Although the resolution of hypercortisolaemia was incomplete and the diagnosis of PPNAD suggested bilateral disease, the patient did not give consent to right adrenalectomy. No other components of Carney complex (CNC) were discovered. Since then, cushingoid features were periodically florid (Fig. 1A). Despite the disease, between 2012 and 2017 the patient gave birth to three healthy children from four spontaneous pregnancies, while only one ended in miscarriage in the $12^{\text {th }}$ week of pregnancy. Finally, being able to undergo concluding hormonal evaluation in 2018, surprisingly, laboratory and radiological findings were consistent with ACTH-dependent CS. Pituitary magnetic resonance imaging (MRI) showed a lesion of less than $3 \mathrm{~mm}$ on the left side of the adenohypophysis (suggesting microadenoma) (Fig. 1B). The results of bilateral inferior petrosal sinus sampling (with ACTH levels much higher in inferior petrosal sinuses than in peripheral circulation) confirmed the diagnosis (Fig. 1C). The patient underwent transsphenoidal selective adenectomy and has been eucortisolaemic for two years since the operation. The genetic testing for PRKAR1A (protein kinase A regulatory 1-alpha subunit) gene mutations was negative, and the second histopathological evaluation (Fig. 1D) raised some considerable doubts whether the diagnosis of PPNAD was stated correctly.

Cushing's syndrome (CS) is characterised by a chronic excess of cortisol, with pituitary adenoma (corticotropinoma) being the most common endogenous source. Adrenal pathologies account for the minority of cases. The diagnostic process to unveil the underlying cause of CS is often challenging. Difficulties can be caused by atypical manifestation of the disease or its periodic nature with hypercortisolaemia alternated with spontaneous resolutions (so-called cyclic CS). However, both PPNAD and corticotropinoma can manifest like this [1]. PPNAD occurs sporadically or more often as part of a familial syndrome called Carney complex, with loss-of-function germline mutations in the PRKAR1A gene as the main underlying genetic reason. Pituitary lesions are not rare in $\mathrm{CNC}$; however, among these tumours just single cases of corticotropinomas have been described only recently $[2,3]$.

In our case, the first diagnosis of ACTH-independent CS was based on scarce biochemical data. Adrenocorticotropin levels were in the lower part of the normal range, and the high-dose dexamethasone test did not give a paradoxical response characteristic for PPNAD. Pituitary MRI was not performed at that time, and hyperandrogenism corresponded poorly to the diagnosis of PPNAD and indicated rather a long ACTH stimulation.

To conclude, this case report points out the importance of careful, repeated evaluation of CS patients. It also enhances the doubts whether prompt, even one-step bilateral adrenalectomy is always the right therapeutic option in suspicion of PPNAD [4]. 


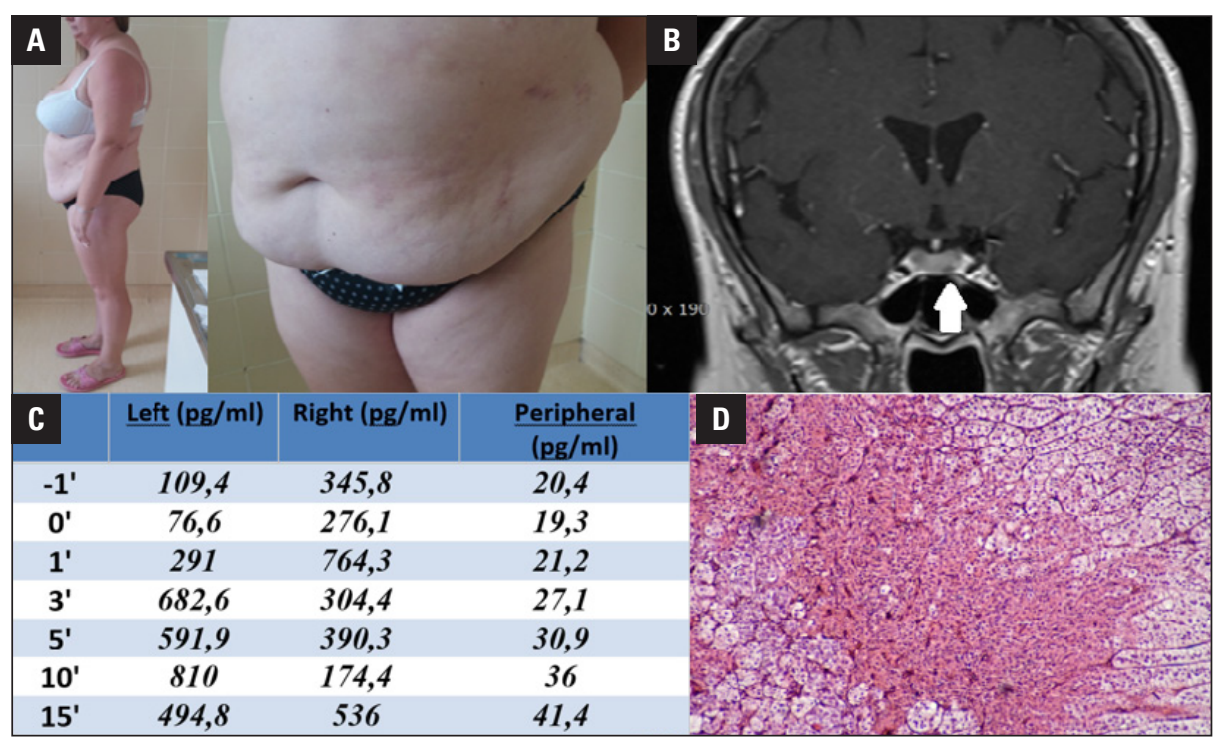

Figure 1. A. Cushingoid features in a 32-year-old female postpartum. B. Gadolinium-enhanced coronal MRI scan of the head showing a microcorticotropinoma pituitary adenoma (white arrow). C. Results of bilateral inferior petrosal sinus sampling (BIPSS) show higher ACTH concentrations from inferior petrosal sinuses than from peripheral vein. D. Histopathological image of equivocal pigmented adrenal hyperplasia (haematoxylin and eosin, $5 \times$ )

\section{Conflict of interest/funding sources}

None declared.

\section{References}

1. Meinardi JR, Wolffenbuttel BHR, Dullaart RPF. Cyclic Cushing's syndrome: a clinical challenge. Eur J Endocrinol. 2007; 157(3): 245-254, doi: 10.1530/EJE-07-0262, indexed in Pubmed: 17766705.
2. Kiefer FW, Winhofer Y, Iacovazzo D, et al. mutation causing pituitary-dependent Cushing disease in a patient with Carney complex. Eur J Endocrinol. 2017; 177(2): K7-KK12, doi: 10.1530/EJE-17-0227, indexed in Pubmed: 28522647

3. Hernández-Ramírez LC, Tatsi C, Lodish MB, et al. Corticotropinoma as a Component of Carney Complex. J Endocr Soc. 2017; 1(7): 918-925, doi: 10.1210/js.2017-00231, indexed in Pubmed: 29264542.

4. Sarlis NJ, Chrousos GP, Doppman JL, et al. Primary pigmented nodular adrenocortical disease: reevaluation of a patient with carney complex 27 years after unilateral adrenalectomy. J Clin Endocrinol Metab. 1997; 82(4): 1274-1278, doi: 10.1210/jcem.82.4.3857, indexed in Pubmed: 9100606. 